\title{
MAGNETIC FIELD EFFECT ON ELECTRODEPOSITION OF COBALT DENDRITES
}

\author{
R. Ballou, P. Molho, S. Pairis \\ Institut Néel, CNRS et Université Joseph Fourier, \\ BP 166, F-38042 Grenoble Cedex 9, France
}

\begin{abstract}
We are studying the effect of a magnetic field on cobalt dendrites grown by electrodeposition in a thin cell. Several electrochemical parameters like $\mathrm{pH}$, current density, initial concentration are known to affect the crystalline structure of electrodeposited cobalt. For two values of $\mathrm{pH}$, we analyze the effect of a high magnetic field $(6 \mathrm{~T})$ on the growth direction of the dendrites with respect to the crystalline axis of the structure.
\end{abstract}

Introduction. We are interested in the effect of a magnetic field and other electrochemical parameters on the nanostructuration, crystallization, grains or crystallites size of materials produced by electrodeposition. A model system for such studies, allowing to get some insight of the basic mechanisms, is thin cell electrodeposition. It leads to arborescent morphologies [1-3], and growth conditions can be varied to amplify the effects of relevant parameters. It is well suited for in situ observations during the growth, at different scales, in order to study the first steps of the growth and the branching phenomena. We are studying especially the growth of magnetic materials, since the effect of a magnetic field may depend on magnetic interactions: the case of Fe has shown spectacular effects on the morphology [4].

The present study is concerned with Co dendrites and the effect of a magnetic field on the growth processes. The properties of the dendrites themselves are also of interest, since the morphology depends strongly on the growth parameters, and then eventually the crystallographic structure and the magnetic properties. Such analysis of the effect of the electrochemical parameters on the growth of cobalt have already been done in some cases, like electrodeposited films, nanowires in membranes and electrodeposited aggregates in thin cells. The present study makes an attempt to analyze the effect of a high magnetic field applied during the growth of Co arborescences electrodeposited in thin cells.

1. Films and nanowires. The crystallographic structure of Co is very sensitive to the electrochemical parameters. Goddard et al. describe the effect of the solution $\mathrm{pH}$ and of a magnetic field applied during the growth of cobalt films electrodeposited on a single crystalline $\mathrm{Cu}$ substrate [5]. When the $\mathrm{pH}$ is lower than 2.4 , the crystalline structure of the Co film is cubic fcc $(\beta \mathrm{Co})$, oriented like the fcc structure of the copper substrate. This structure is usually stable only above $425^{\circ} \mathrm{C}$. When the $\mathrm{pH}$ is greater than 2.9 , the crystalline structure of the Co film is hexagonal hcp $(\alpha \mathrm{Co})$. For an intermediate value of $\mathrm{pH}$, there is a mixing of phases. When a magnetic field of $1.7 \mathrm{~T}$ is applied in the plane of growth (whatever its orientation), it reduces the $\mathrm{pH}$ range for the phase mixing.

In the case of nanowires grown in membranes [6,7], the crystalline structure may be cubic or hexagonal. In the latter case, the $c$-axis may be either parallel or perpendicular to the wire axis, depending on the $\mathrm{pH}$ of the electrolyte and on the 
current density during growth. In experiments described by Tourillon et al. [6], depending on the pulsed electrodeposition parameters (namely, the overpotential used to initiate the growth), the structure may be a pure hexagonal phase, or a polytype structure, stacking of hcp and fcc. Darques et al. [7] studied nanowires in polycarbonate membranes. The nanowires are usually polycrystalline, each grain having a hcp structure, with the $c$-axis at few degrees from the perpendicular to the wire axis. In fact, different orientations of the $c$-axis with respect to the wire axis are observed, depending on the $\mathrm{pH}$. For a $\mathrm{pH}$ larger than 6 , the $c$-axis is along the wire axis. For a $\mathrm{pH}$ lower than 5 , the $c$-axis is perpendicular to the wire axis. For intermediate values of $\mathrm{pH}$, the axis orientation depends on the current, $c$ is parallel to the wire axis in low currents and perpendicular to the wire axis in high currents. The coexistence of hcp and fcc is also observed.

With a dendrite being a system intermediate between a film (2D) and a wire (1D), one could expect the same kind of behaviour, namely, an effect of $\mathrm{pH}$ and magnetic field on the crystalline structure. In particular, a magnetic field seems to be a parameter able to modify and adjust the anisotropy, which is an interesting issue in terms of monitoring the growth processes in electrodeposition.

2. Electrodeposited Co dendrites. We had performed some previous experiments of electrodeposition of Co in thin cells [8]. In fact, the cells were open, their thickness, being defined by the anode thickness, was $200 \mu \mathrm{m}$. The electrolytic solution of $\mathrm{CoSO}_{4}$ in water (concentration 0.5 mole/l) had an initial pH of 4. Magnetic fields were applied either in the plane of growth (up to $1.2 \mathrm{~T}$ ) or perpendicular to the plane of growth (up to $0.2 \mathrm{~T}$ ), using a permanent magnets device. Under such experimental conditions, the growth is always dendritic. When no magnetic field is applied, the crystalline structure is hcp, with the $c$-axis being perpendicular to the plane of growth. The diffraction pattern of a small dendrite grown with no field (Fig. 1a) reveals its hexagonal structure, with the $c$-axis being perpendicular to the plane of growth (the dendrite is flat, and the electron beam is perpendicular to its plane).

When a magnetic field is applied parallel to the plane of growth, the macroscopic morphology is elongated along the field, but at the TEM scale there is no signature of the magnetic field, and the $c$-axis remains perpendicular to the plane

(a)

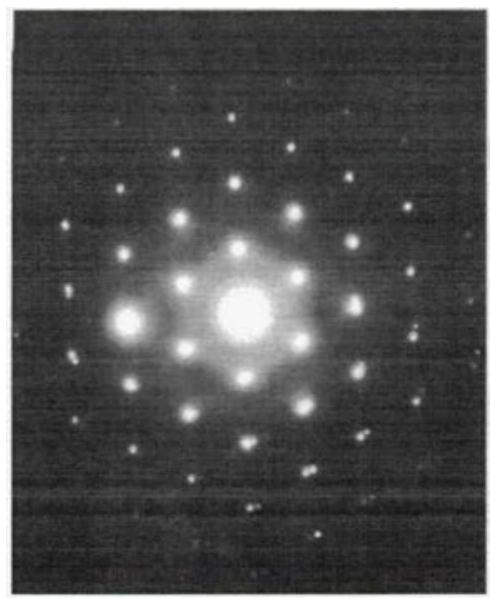

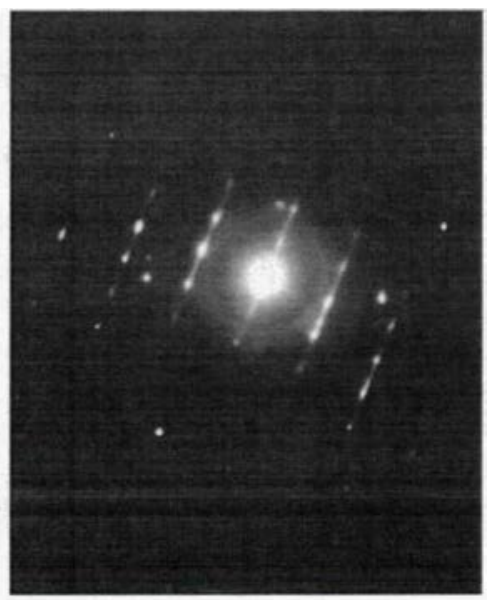

$(b)$

Fig. 1. Diffraction pattern of Co dendrites (from [8]). (a): no field, zone axis [001] of the hcp structure; $(b): H=0.2 \mathrm{~T}$ applied perpendicular to the plane of growth. 
of growth. When a field of $0.2 \mathrm{~T}$ is applied normal to the plane of growth, the first point is that there is no spiraling, as would be expected from Lorentz forces. This can result, like in the case of iron, from some rigidity of the metal itself or due to its magnetization in the field. The second point is that the $c$-axis of the hcp structure appears now to be in the plane of growth, as revealed by the diffraction pattern (Fig. 1b). The lines in the diagram result from defects in the structure interpreted as extended inclusions of the fcc phase into hcp. The effect of the magnetic field normal to the plane of growth is then a flip of the $c$-axis of the crystalline structure with respect to the growth axis.

Transmission Electron Microscopy (TEM) observations and the corresponding diffraction patterns were realized on very small dendrites. In order to better characterize this axis flip, we have performed new experiments under more controlled conditions, in particular, concerning the $\mathrm{pH}$ of the solution, and using a high magnetic field up to $6 \mathrm{~T}$. Observations at macroscopic scale are realized by optical imaging during the growth and the ones at small scale by means of Scanning Electron Microscopy (SEM), allowing to study bigger and thicker dendrites than those observed by TEM. The microscope we used is a ZEISS-Ultra+ FEG-SEM, Field Effect Gun-Scanning Electron Microscope.

3. Present experiments. The growth conditions are the following: the cell thickness is $100 \mu \mathrm{m}$, the initial concentration is $0.5 \mathrm{~mole} / \mathrm{l}$, and the applied voltage, constant during the growth, is $5 \mathrm{~V}$. The experiments are done for two values of $\mathrm{pH}: \mathrm{pH}=2$ is obtained by adding boric acid, $\mathrm{H}_{3} \mathrm{BO}_{3}$, to the solution (in order to stabilize the $\mathrm{pH}$ ) and few droplets of $\mathrm{H}_{2} \mathrm{SO}_{4} ; \mathrm{pH}=4$ is obtained either using only $\mathrm{H}_{2} \mathrm{O}$, in order to reproduce the same conditions as in the previous experiment, or by adding $\mathrm{H}_{3} \mathrm{BO}_{3}$ to the solution. The magnetic field, $6 \mathrm{~T}$, is applied perpendicular to the cell.

3.1. Macroscopic observations (Fig. 2). Comparing the two cases with boric acid in zero field, the aggregates seem thicker at $\mathrm{pH}=4$ than at $\mathrm{pH}=2$. The growths start with no $\mathrm{H}_{2}$ bubbles, but after some time bubbles appear, perturbing the growth at large scale. The morphology at small scale, however, observed optically, seems not affected. The effect of the $6 \mathrm{~T}$ field in both cases $(\mathrm{pH}=2$ and 4 ) is a thinning of the branches and more $\mathrm{H}_{2}$ bubbles production. Under such growth conditions, the magnetic field seems not to facilitate the growth and the aggregate quality, at least when observed at this macroscopic scale. With the field being perpendicular to the cell, the Lorentz forces induce a global convection in the cell that was observed, but that is not pushing or breaking the growing branches. In the case without sulfuric or boric acid, giving an initial $\mathrm{pH}$ of 4 , the effect of the field is somehow different. The growth starts with no bubbles production, as a regular growth of dendrites well ramified, but soon the growth stops completely inhibited by the appearance of $\mathrm{H}_{2}$ bubbles, leaving a very small aggregate.

To summarize, in all cases the growths are dendritic, the aggregates are not very dense, the secondary branches are quite small and $\mathrm{H}_{2}$ bubble formation perturbs the growth. The effects of changing the $\mathrm{pH}$ and applying a magnetic field are not very pronounced and do not lead to obvious structural modification of the cobalt dendrites. We can only notice that under the field the branches seem to be thinner. There is no spiraling of the aggregates grown under a magnetic field perpendicular to the cell, even if the convection induced by the Lorentz body force is present and visualized by particles moving inside the solution. Finally, one observes an inhibition of the growth under the field in the case when no boric acid is added to the electrolyte. 
OT

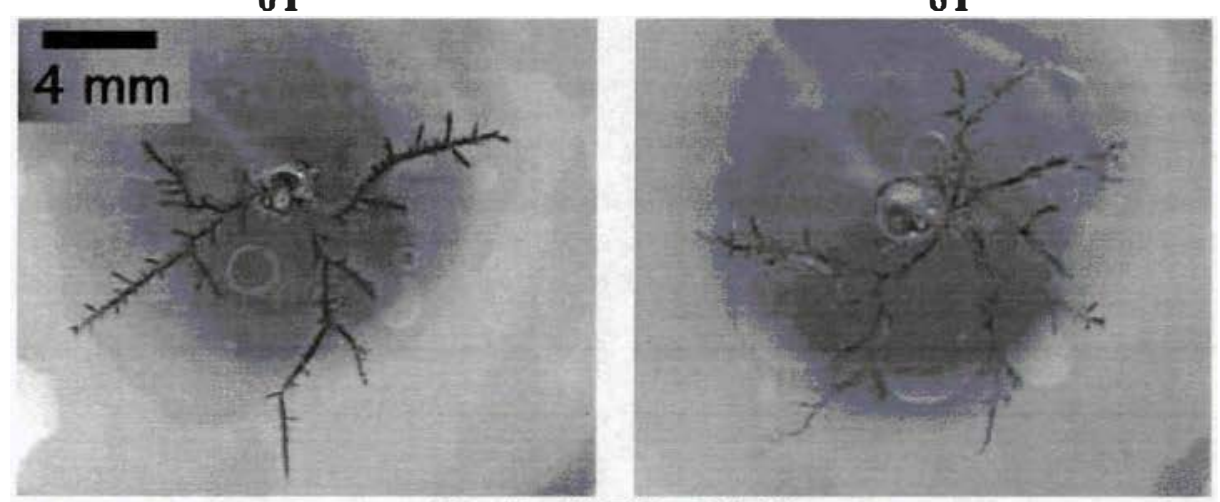

$\mathrm{pH}=2-\mathrm{H}_{3} \mathrm{BO}_{3}+\mathrm{H}_{2} \mathrm{SO}_{4}$
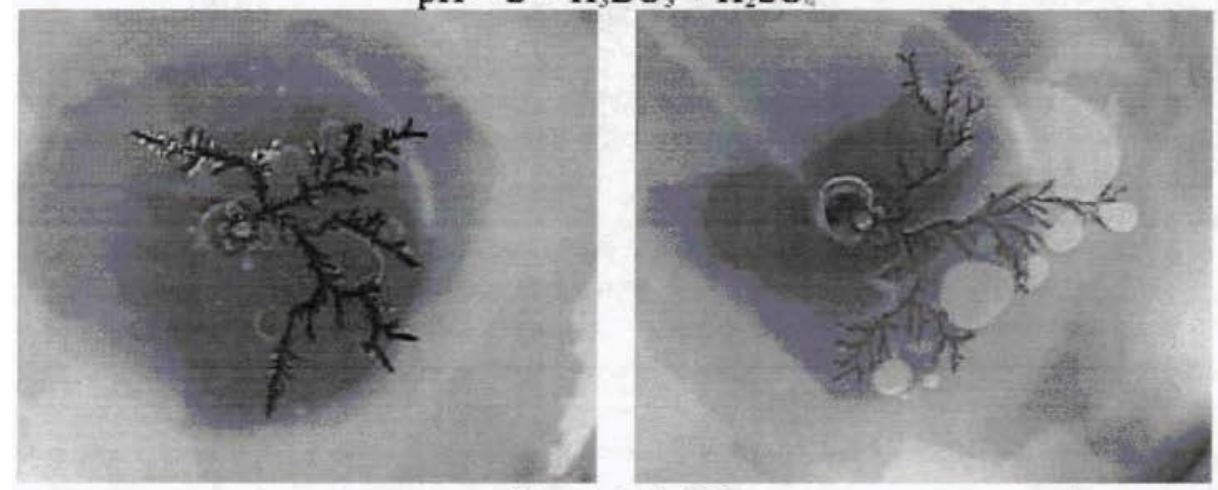

$\mathrm{pH}=4-\mathrm{H}_{3} \mathrm{BO}_{3}$
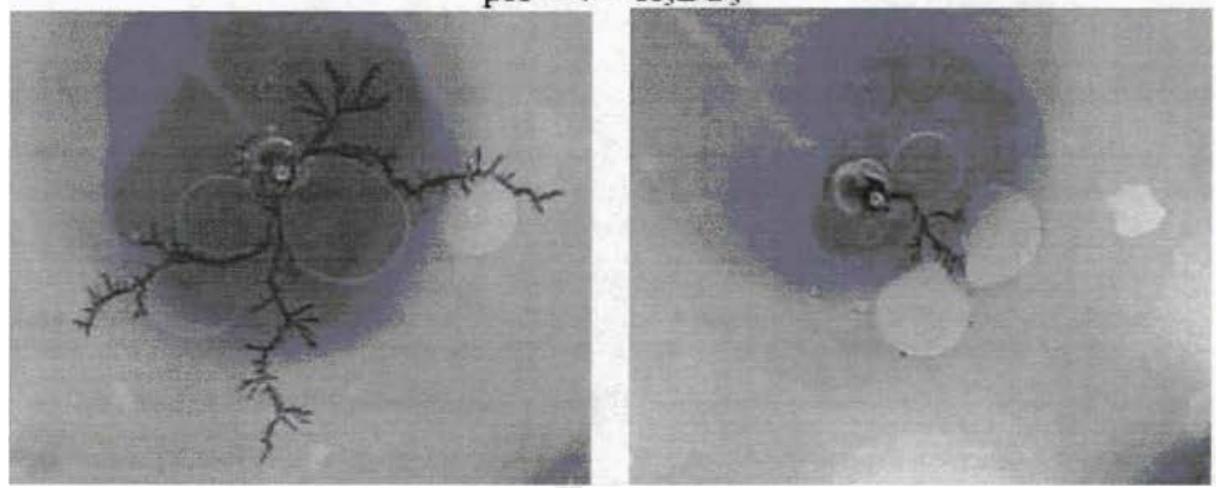

$$
\mathrm{pII}=4-\mathrm{I}_{2} \mathrm{O}
$$

Fig. 2. Macroscopic morphologies of Co dendrites as a function of $\mathrm{pH}$ and magnetic field. Initial concentration 0.5 mole/l, applied voltage $5 \mathrm{~V}$.

3.2. Small scale observations. In order to get structural information, we did SEM observations. Of course these observations cannot be done in situ during the growth. The first step is then to collect the dendrites after the growth. Opening the cell leads to a destruction of the large scale features, but small sizes are not affected, even if disorder is introduced compared to the macroscopic organization. Water is added to eliminate the salt, which could remain on the floating aggregates, then the dendrites are collected and dried onto a piece of paper. Finally, they are transferred onto a sticking conducting carbon tape for electron microscopy. 
Three types of characterization are possible with electron microscopy: structure, using Electron Backscatter Diffraction (EBSD), morphology with SEM images, and chemical composition with Energy Dispersive X-rays spectroscopy (EDX).

EBSD allows to get direct structural information, but in our case, it was unsuccessful, due to the irregularity of the samples, implying that there is very a little chance that diffracted electrons reach the detector. EBSD is performed at $20 \mathrm{kV}$ and tilting the sample by $70^{\circ}$. Since the topography of the dendrites is rough, there is a screening of the primary electrons beam, but also of the backscattered electrons, which in most cases prevent from measuring the orientation of the dendrites. We observed only one positive case, an isolated tip of a quite thick dendrite grown at $\mathrm{pH}=4$ and $H=0$. The obtained diagram leads to an indexation more likely compatible with the cubic phase. These observations are then not conclusive, and EBSD seems not adapted to irregular samples (results much better are obtained with films).

SEM images are obtained with a $20 \mathrm{kV}$ voltage, collecting the secondary electrons. Images were analyzed for different growth conditions to characterize $\mathrm{pH}$ and magnetic field effects on the morphology and crystalline structure. In all cases, the growth is clearly dendritic, but, having no diffraction pattern, it is not possible to characterize the crystalline structure directly and relate the growth direction (the dendrite axis) to the crystalline axis. The information about the structure is then obtained only by the observation of the angles between the branches in the $\mathrm{SEM}$ images. Images of dendrites grown from solutions with $\mathrm{pH}=2$ and 4 and under magnetic fields $H=0$ and $6 \mathrm{~T}$ are presented in the following figures.

- $\mathrm{pH}=2, H=0$. Fig. $3 a$ presents an image of a very typical dendrite grown under such conditions. It is well crystallized, almost $2 \mathrm{D}$ at large scale and with small crystallites on top of the branches. It reveals a hcp structure, with the $c$-axis perpendicular to the growth plane, since the angle between the branches is about $120^{\circ}$. A closer view of the small crystallites (Fig. $3 b$ ) shows that they seem to be small single crystals, not ramified. Fig. $3 c$ illustrates one case, quite rare, with a cubic symmetry, where the angle between the branches is close to $90^{\circ}$. This could mean that this dendrite has a fcc structure, or that it has a hcp structure with the $c$-axis in the plane of growth.

- $\mathrm{pH}=2, H=6 \mathrm{~T}$. Fig. 4 presents images of a dendrite at different scales. The dendrite is again $2 \mathrm{D}$, still the hcp structure with the $c$-axis perpendicular to the growth plane, and with crystallites on top of the branches (Fig. 4a). Fig. $4 b$ presents a very small dendrite, showing that ramifications exist down to the $100 \mathrm{~nm}$ scale. In that sample, EDX characterization is performed in order to check the chemical composition of different objects observed in the image, dendrites of dif-

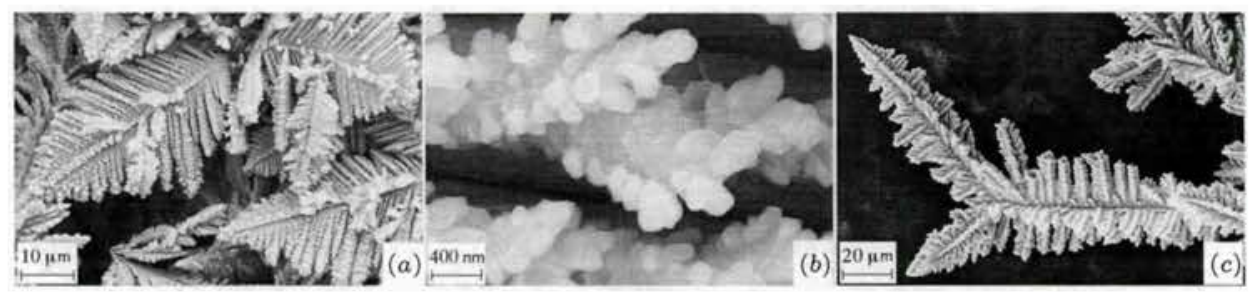

Fig. 3. $\mathrm{pH}=2, H=0$, SEM images. (a): typical dendrites; (b): small crystallites on the top of the branches; $(c)$ : quite a rare case of a dendrite with $90^{\circ}$ angle between the branches, revealing either a fcc structure or a hcp structure with the $c$-axis within the growth plane. 

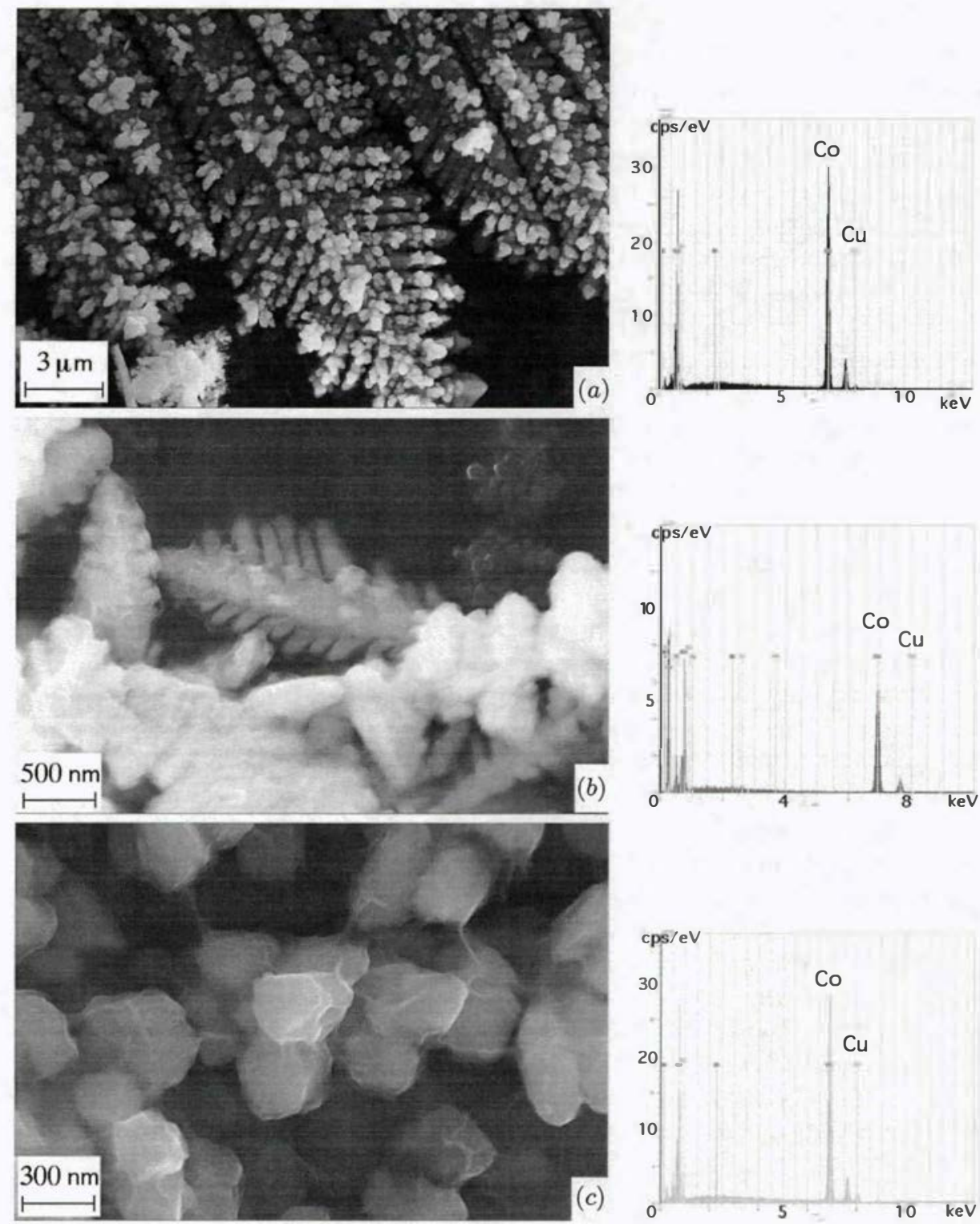

Fig. 4. $\mathrm{pH}=2, H=6 \mathrm{~T}, \mathrm{SEM}$ images and corresponding EDX characterization of different objects. (a): a large dendrite; $(b)$ : a very small dendrite, showing that ramifications exist down to the $100 \mathrm{~nm}$ scale; $(c)$ : small crystallites.
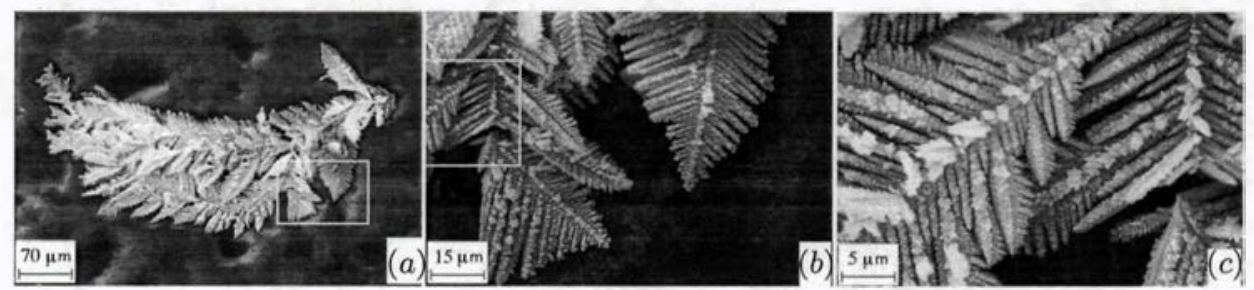

Fig. 5. $\mathrm{pH}=4$ (with boric acid), $H=0$, SEM images at different scales. (b) and (c) are enlarged views, each corresponding to the square in the previous image. 


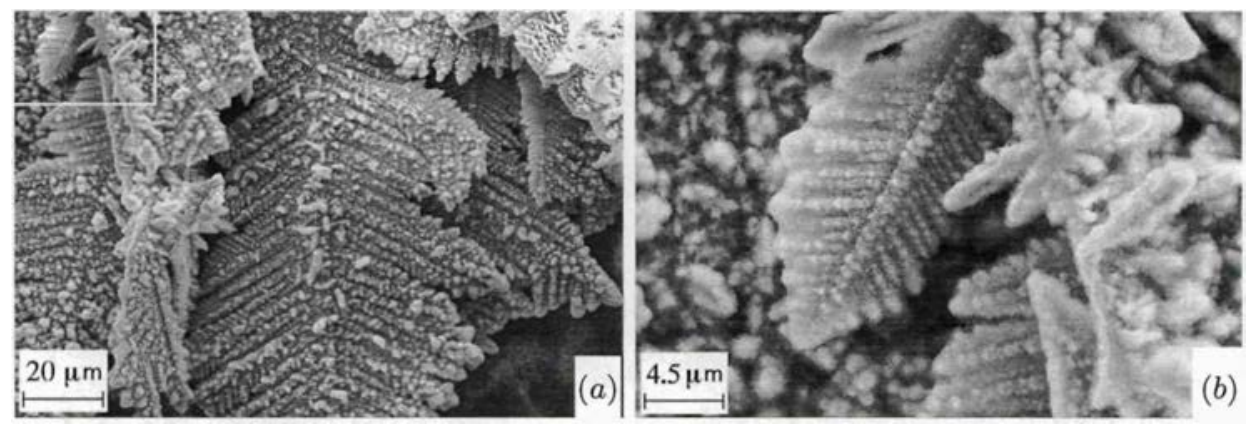

Fig. 6. $\mathrm{pH}=4$ (with boric acid), $H=6 \mathrm{~T}$, SEM images at two different scales.

ferent size and crystallites on the top of the branches. The result of this analysis is that all objects are mainly $\mathrm{Co}$, with a small amount of $\mathrm{Cu}$ in large dendrites and in crystallites, originating from the dissolution of the anode during the growth.

- $\mathrm{pH}=4, H=0$. The same kinds of images are obtained. Fig. 5 presents a zoom from a large dendrite to see the relation between the different scales. The crystalline structure is still hcp, with the $c$-axis perpendicular to the growth plane, since the angle between the branches is about $120^{\circ}$.

- $\mathrm{pH}=4, H=6$ T. Fig. 6 presents images at two scales: again the angle between the branches is about $120^{\circ}$, suggesting a hcp structure with the $c$-axis perpendicular to the growth plane.

Conclusion. The main conclusion of these observations is that no real effect of the magnetic field on the crystalline structure of cobalt dendrites is observed: no spiraling, even under $6 \mathrm{~T}$, and when the global MHD convection induced by the Lorentz body force is observed. No flip of the $c$-axis of the hcp structure from perpendicular to parallel to the growth plane. The effect of the field may just consist in slight morphology changes, affecting the shape of the dendrites. If some structural changes could be induced by a magnetic field, it should be under conditions, where the structure is already unstable, for instance, due to $\mathrm{pH}$ values close to a "threshold" for structural modifications. When the $\mathrm{pH}$ is 2 or 4 , the growth conditions are too stable, apart maybe at the tip of slowing down dendrites, when the tip become thicker and the $\mathrm{pH}$ conditions change locally.

Acknowledgements. High field experiments were performed at CRETA, CNRS, Grenoble.

\section{REFERENCES}

[1] M. Matsushita, M.Sano, Y. Hayakawa, H. Honjo, Y. Sawada. Fractal structures of zinc metal leaves grown by electrodeposition. Phys. Rev. Lett., vol. 53 (1984), pp. 286-289.

[2] D. Grier, E. Ben-Jacob, R. Clarke, L.M. Sander. Morphology and microstructure in electrochemical deposition of zinc. Phys. Rev. Lett., vol. 56 (1986), pp. 1260-1263.

[3] Y.Sawada, A. Dougherty, J.P. Gollub. Dendritic and fractal patterns in electrolytic metal deposits. Phys. Rev. Lett., vol. 56 (1986), pp. 1264-1267. 
[4] S. Bodea, L. Vignon, R. Ballou, P. Molho. Electrochemical growth of iron arborescences under in-plane magnetic field: Morphology symmetry breaking. Phys. Rev. Lett., vol. 83 (1999), pp. 2612-2615.

[5] J. Goddard, J.G. Wright. The effect of solution $\mathrm{pH}$ and applied magnetic field on the electrodeposition of single-crystal films of cobalt. Brit. J. Appl. Phys., vol. 15 (1964), pp. 807-815.

[6] G. Tourillon, L. Pontonnier, J.P. Levy, V. Langlais. Electrochemically synthesized Co and Fe nanowires and nanotubes. Electrochem. SolidState Lett., vol. 3 (2000), pp. 20-23.

[7] M. Darques, L. Piraux, A. Encinas, P. Bayle-Gulllemaud, A. Popa, U. EBELS. Electrochemical control and selection of the structural and magnetic properties of cobalt nanowires. Appl. Phys. Lett., vol. 86 (2005), pp. 072505-3.

[8] S. Bodea, R. Ballou, L. Pontonnier, P. Molho. Electrochemical growth of iron and cobalt arborescences under a magnetic field: A TEM study. Phys. Rev. B., vol. 66 (2002), pp. 224104-6. 